\title{
Potential Health Hazards of Additive Manufacturing
}

\author{
Martin Nozar, Vaclava Pokorna \& Ivana Zetkova
}
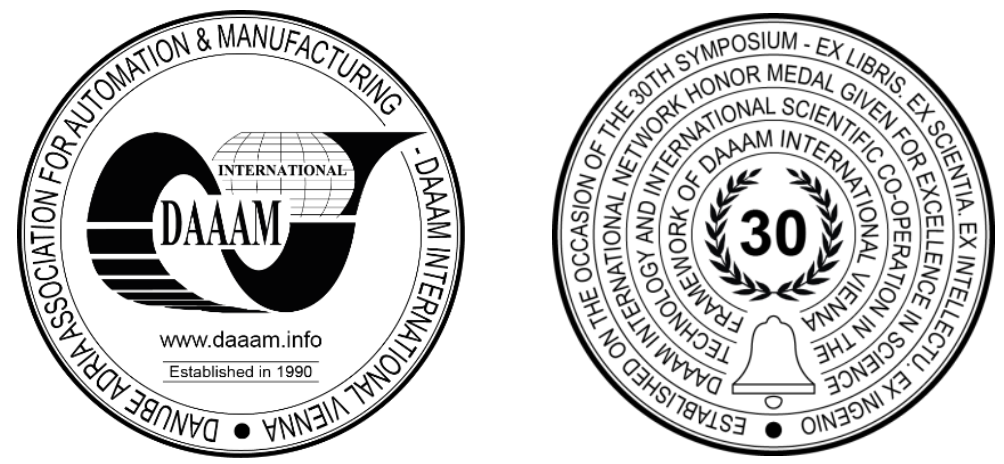

This Publication has to be referred as: Nozar, M[artin]; Pokorna, V[aclava] \& Zetkova, I[vana] (2019). Potential Health Hazards of Additive Manufacturing, Proceedings of the 30th DAAAM International Symposium, pp.0654-0662, B. Katalinic (Ed.), Published by DAAAM International, ISBN 978-3-902734-22-8, ISSN 1726-9679, Vienna, Austria DOI: $10.2507 / 30$ th.daaam.proceedings.090

\begin{abstract}
In recent years, additive manufacturing has begun to penetrate not only into a number of industrial enterprises, but also into households. 3D printers are becoming more affordable and appear on the market in a variety of designs that use a variety of principles and feedstock materials. However, this advanced technology brings not only a unique manufacturing potential but also a number of hazards for its users as well as for their surroundings and the environment. Unfortunately, these hazards are often unsuspected and can easily be underestimated or neglected. The aim of this article is therefore to map available additive technologies, to point out the hazards involved and their possible consequences and to suggest possible ways of avoiding or mitigating these hazards as much as possible. The article describes processes connected with the greatest hazards in metal additive manufacturing, which is the most dangerous in terms of the number and nature of the hazards involved.
\end{abstract}

Keywords: Additive manufacturing; AM machine; metal powder; health hazards; protective equipment.

\section{Introduction}

\subsection{Additive manufacturing}

Additive manufacturing (AM), also known as 3D printing, rapid manufacturing or additive fabrication is defined by the American Society for Testing and Materials (ASTM) [9] as "The process of joining materials to make objects from 3D model data, usually layer upon layer, as opposed to subtractive manufacturing methodologies".

Additive manufacturing has witnessed intensive development in recent years and has succeeded in penetrating into a number of branches of industry where they are used to produce various components and objects not only from plastic, paper or concrete, but also from composite materials or metals. In most cases, such products have the same or even better properties than their predecessors made by conventional building, machining or forming technologies. Although this technology can create very complicated shapes and structures which are almost impossible to manufacture using traditional production processes, these technologies still have their own limitations and present new and dangerous health hazards for their operators and for the environment [10]. 
The feedstock material for most plastic or metal additive manufacturing technologies is a special and very fine powder which itself means an unnoticeable but serious hazard. This powder consists of spherical particles of different sizes, of which the smallest ones are invisible to the naked eye and are able to penetrate virtually anywhere; unfortunately, including the human body. These particles are carcinogenic, and a cloud composed of them is in certain conditions prone to fire or explosion (i.e. especially aluminium powder). Nonetheless, very small metal particles or residues do not occur only in additive manufacturing but also arise during conventional manufacturing, especially grinding, although in smaller quantities and concentrations. In any case it is essential to pay very good attention to such extremely small metal or plastic particles and protect ourselves, our colleagues, employees or visitors and, of course, the environment, from their harmful effects.

\subsection{Comprehensive research of additive manufacturing}

Comprehensive research into all aspects of additive manufacturing is conducted at the Experimental Machining Laboratory of the Regional Technological Institute in the University of West Bohemia's Faculty of Mechanical Engineering. This laboratory specializes in designing and manufacturing advanced cutting tools, including preparation of their microgeometry, detailed measurements and grinding and polishing of sculptured surfaces. These cutting tools are manufactured either by conventional machining or by additive manufacturing. For this purpose and for manufacturing special prototypes as well, the laboratory uses an advanced metal AM machine (a 3D printer). Small metal particles and residues are present during such machining and manufacturing processes and therefore the laboratory pays considerable attention to the research of these dangerous particles and materials as well as to the assessment and development of proper protective equipment.

\section{Categories of Additive Manufacturing and materials used}

The hazards caused by additive manufacturing depend primarily on the actual printing technology principle, on feedstock materials used and on ways of handling them and the machine. The American Society for Testing and Materials (ASTM) has created a comprehensive classification of additive manufacturing technologies according to this principle [9], [8].

\subsection{Vat Photopolymerisation}

Vat photopolymerisation uses a vat of liquid photopolymer resin, out of which the model is constructed layer by layer. An ultraviolet light is used to cure or harden the resin where required, whilst a platform moves the object being made downwards after each new layer is cured.

Materials used: Polymers (e.g. UV-curable photopolymer resin), Resins (e.g. Visijet range)

\subsection{Material Extrusion}

Material is drawn through a nozzle, where it is heated and is then deposited layer by layer. The nozzle can move horizontally and a platform moves up and down vertically after each new layer is deposited.

Materials used: Polymers (e.g. ABS, Nylon, PC, PC, AB)

\subsection{Sheet Lamination}

The Ultrasonic Additive Manufacturing process uses sheets or ribbons of metal, which are bound together using ultrasonic welding. The process does require additional CNC machining and removal of the unbound metal, often during the welding process. Alternative technology called Laminated Object Manufacturing uses a similar layer by layer approach but uses paper as material and adhesive instead of welding.

Materials used: Paper, Plastic, Sheet metals

\subsection{Directed Energy Deposition}

A typical DED machine consists of a nozzle mounted on a multi axis arm, which deposits melted material onto the specified surface, where it solidifies. The material, which can be deposited from any angle using 4 and 5 axis machines, is melted upon deposition with a laser or electron beam.

Materials used: Metals (e.g. Cobalt Chrome, Titanium), Polymers, Ceramics 


\subsection{Binder Jetting}

The binder jetting process uses two materials: a powder-based material and a binder. The binder acts as an adhesive between powder layers. The binder is usually in liquid form and the build material in powder form. A print head moves horizontally along the $\mathrm{x}$ and $\mathrm{y}$ axes of the machine and deposits alternating layers of the build material and the binding material. After each layer, the object being printed is lowered on its build platform.

Materials used: Metals (e.g. Stainless steel), Polymers (e.g. ABS, PA, PC), Ceramics, Glass

\subsection{Material Jetting}

Material is jetted onto the build surface or platform, where it solidifies and the model is built layer by layer. Material is deposited from a nozzle which moves horizontally across the build platform. The material layers are then cured or hardened using ultraviolet light. In the case of Nano Particle Jetting, where material dispersions of liquid and metal or ceramic droplets is jetted; the pre-sintered parts are processed in a sintering furnace.

Materials used: Polymers (e.g. Polypropylene, HDPE, PS, PMMA, PC, ABS, HIPS, EDP), Metals, Ceramics (e.g. Zirconia), Wax

\subsection{Powder Bed Fusion}

Powder bed fusion methods use either a laser or electron beam to melt and fuse material powder together. All processes involve the spreading of the powder material (a hopper or a reservoir below or beside the bed provides fresh material supply) over previous layers. Layers are added with a roller or a blade in between fusion (sintering) of individual layers. Electron beam melting requires a vacuum.

Materials used: Metals (e.g. Stainless Steel, Titanium, Aluminium, Cobalt Chrome), Polymers (e.g. Nylon)

\section{Feedstock material handling}

The powder used in additive manufacturing can be of various compositions, sizes and shapes. Metal and plastic powder is a basic feedstock for some additive manufacturing technologies, but some particles can also arise during the manufacturing process. Such particles, which may be of different compositions and even smaller in size than the original ones, can degrade print quality and be dangerous to the AM machine operator. Metal powder (e.g. aluminium) exhibits the greatest number of risk aspects, and therefore its characteristics, manufacturing processes and working with metal powders will be described in this paper.

\subsection{Metal powder manufacturing}

The mechanical properties of metal powder are determined by the manufacturing process. The major manufacturing processes for AM powders are Gas Atomisation, Electrode Induction Melting Gas Atomization (EIGA), Plasma Atomised Wire (PAW) or Plasma Rotating Electrode Process (PREP) followed by water atomisation, crushing and spheroidising, and precipitation from chemical solutions and gas phases.

Gas Atomisation is common and, in many cases, the best technology for producing high quality powder at a reasonable cost. In this process, a molten metal stream is disintegrated into droplets by a high-pressure gas stream. The drops free fall inside a tower and solidify before collection. During free fall, the surface tension of the metal has time to pull the drop into a sphere. To protect the metal from oxidation, the atomising gas is usually nitrogen or argon. Powder size can be controlled by adjusting metal flow rate, gas pressure and flow, and nozzle design [3].

The melting method and atomising gas have a significant influence on properties of the powder. The powders are usually tested to find the following data: morphology, chemistry, particle size distribution, apparent density, packing density and Hall flow rate [3].

\subsection{Metal powder specifics}

Although all powder properties are important for additive manufacturing quality, the powder particle sizes are crucial in terms of safety (too small particles are generally problematic and undesirable). The powder producers deliver powder with specific desirable particle size distribution which is usually described in percentiles: D10, D50 and D90. D10 indicates that $10 \%$ of powder by weight is finer than this micron size. The real particle size distribution of Maraging steel MS1 sample is shown in Fig. 1. 

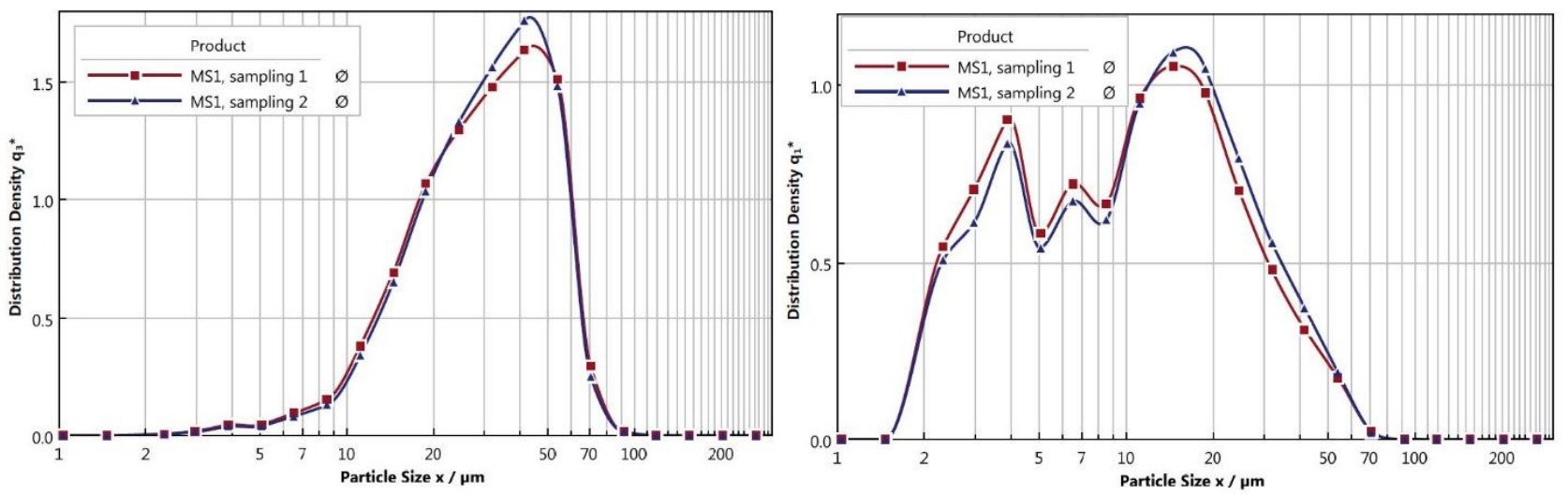

Fig. 1. Density distribution curves q3* and q1*

The curves in Fig. 1 show particle size distributions of the same MS1 powder sample. The commonly used volume curve, which is based on particle volume measurement (Density q3* parameter), effectively hides the fine particles and makes it difficult to see any variations. The basis for understanding a powder is to study the particle count, also called a population. The population curve is dramatically different to the volume curve because it reflects real numbers of particles of various sizes (Density q1* parameter) [3]. This means that particles of small sizes, even though they do not reach high proportions throughout the volume, could be present in large quantities in the powder. For example, in the volume curve, one particle of $50 \mu \mathrm{m}=1000$ particles of $5 \mu \mathrm{m}$ size. And these small particles are the most dangerous and undesirable ones.

Both graphs indicate powder sizes roughly between 10 and $70 \mu \mathrm{m}$, which is a quite common result for a metal powder, nonetheless, particles even smaller than $1 \mu \mathrm{m}$ could be detected around the AM machine. These particles can come from a virgin powder but probably could also arise during the printing process itself. The process of laser melting of metals creates vaporized soot, some of which deposits on the process chamber, and the smoke particles can be even finer than the powder [4].

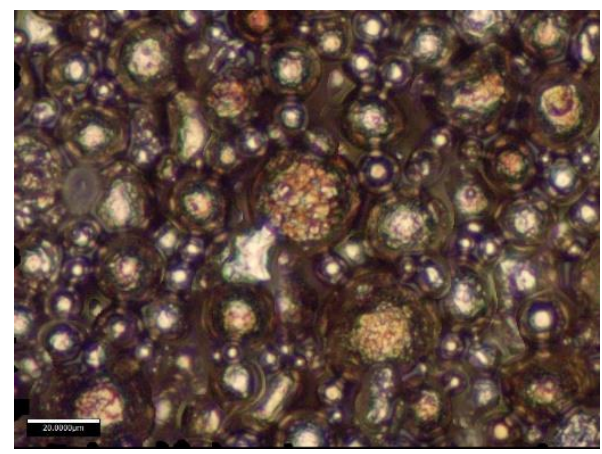

Fig. 2. Enlarged view of MS1 powder particles trapped in support structures (the black line represents $20 \mu \mathrm{m}$ size)

\subsection{Powder handling}

The metal powder during the additive manufacturing process requires human interaction at many steps - from arriving in a container, through multiple recycling steps (unsintered powder is sieved and reused), through to final disposal. The trend in recent years is to minimize this operator interaction and contact with metal powder using advanced technical solutions, where AM machines and auxiliary devices are sealed and perform some operations automatically or at least, enable manipulation of the printing platform and metal powder inside the process chamber using in-built rubber sleeves. However, older AM machines and accessories are not so advanced and equipped, and therefore many operators all around the world come in contact with the powder for long periods every day.

In additive manufacturing, there are several frequently repeated operations when workers could come into contact with the powder:

Pre-processing phase:

- Cleaning the filter module and changing filters in the process gas circuit

- Changing the powder - cleaning the machine and pouring powder into barrels

- Refilling the powder reservoir - pouring powder and filling a dispenser duct

- Upsetting/compacting powder in the dispenser

- Fitting, adjusting a building platform and setting-up a first layer 
Post-processing phase:

- Scooping, wiping and extracting unsintered metal powder off the building platform

- Removing the building platform from the process chamber

- Emptying the collecting duct and sieving the unsintered powder

- Wiping soot residue deposits and cleaning the process chamber

- Cutting printed parts off the platform (the powder is often trapped inside support structures)

- Removing support structures of printed parts (some of them still contain the powder)

- Finishing printed parts (e.g. grinding, shot peening, polishing)

\section{Health hazards of AM machine operation}

As mentioned above, additive manufacturing offers many advantages, but it also raises new considerations for manufacturers and users that include potential safety issues [1]. These issues are associated primarily with the technology itself and feedstock materials used, especially powders of various types and chemical compositions. Although plastic AM technology has its own specifics, metal AM technologies cover most of the hazards associated with plastic printing, and add many other hazards. Therefore, the following hazard overview includes warnings and a list of hazards arising from metal additive manufacturing.

\subsection{Fire and Explosion}

It is essential to operate the AM machine in a safe manner all the time because a potentially explosive atmosphere can be produced in the closed area around the AM machine; the metal powder is flammable and metal condensate is highly flammable. As shown in Figure 3, when handling powder in ambient atmospheres (with oxygen), all that is needed is a suitable ignition source to initiate a fire. Further, if this occurs in the presence of a dust cloud with many particles dispersed in a contained area (such as a small room or an air duct), this could lead to a more damaging explosion [4].
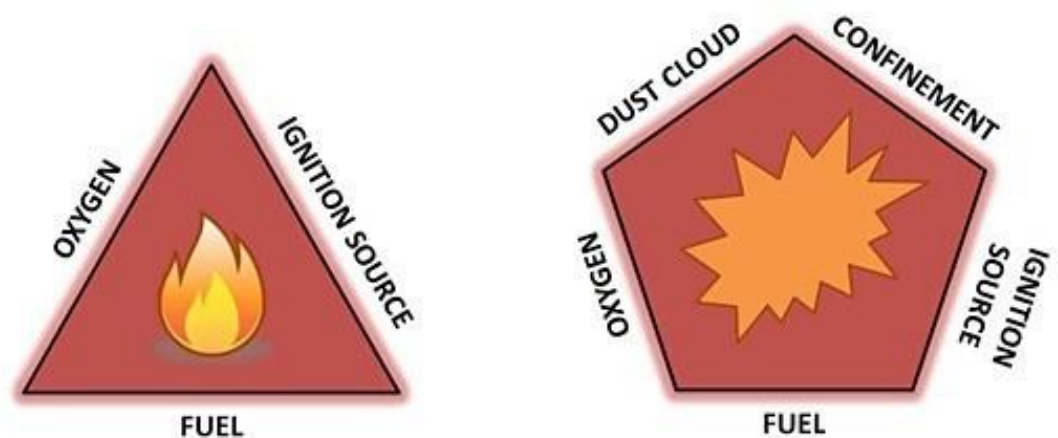

Fig. 3. Fire triangle and explosion pentagon - condition combinations for ignition [4]

Fire: When handling metal powder, the user needs to be aware that she/he has already met two of the three requirements for a fire, and the main aim must be to ensure protection against any ignition source. While there are several sources that could cause ignition, the most likely source for a user of a metal AM machine is static electricity. Additionally, it is possible that a fire could be initiated by hot surfaces, flames, hot gases and particles, mechanically generated sparks, and stray electrical currents [4].

Explosion: With regard to explosions, in addition to the three requirements above, dust clouds in contained areas can exacerbate any ignition to a much larger impact within milliseconds. Therefore, the prevention of the formation of metal dust clouds, as unlikely as that may seem, is of paramount importance [4].

\subsection{Powder inhalation and contact}

The effect of metal particles on human organs is still poorly investigated and documented, and ingestion of fine metal powder is an underestimated occupational health factor [2]. According to current knowledge, there are three ways that powders can interact with the body [5]:

- Skin contact, which may lead to some form of irritation or allergic reaction (e.g. dermatitis). In the case of nanopowders, the particles can penetrate the skin and become absorbed into cells in various parts of the body, including the brain

- Eye contact, resulting in mechanical irritation and cause damage

- Inhalation and either ingestion by swallowing or entering the lungs, resulting in possible breathing problems. 
In the case of inhalation, the size of the particles determines the level of penetration into the lungs (Fig. 4).

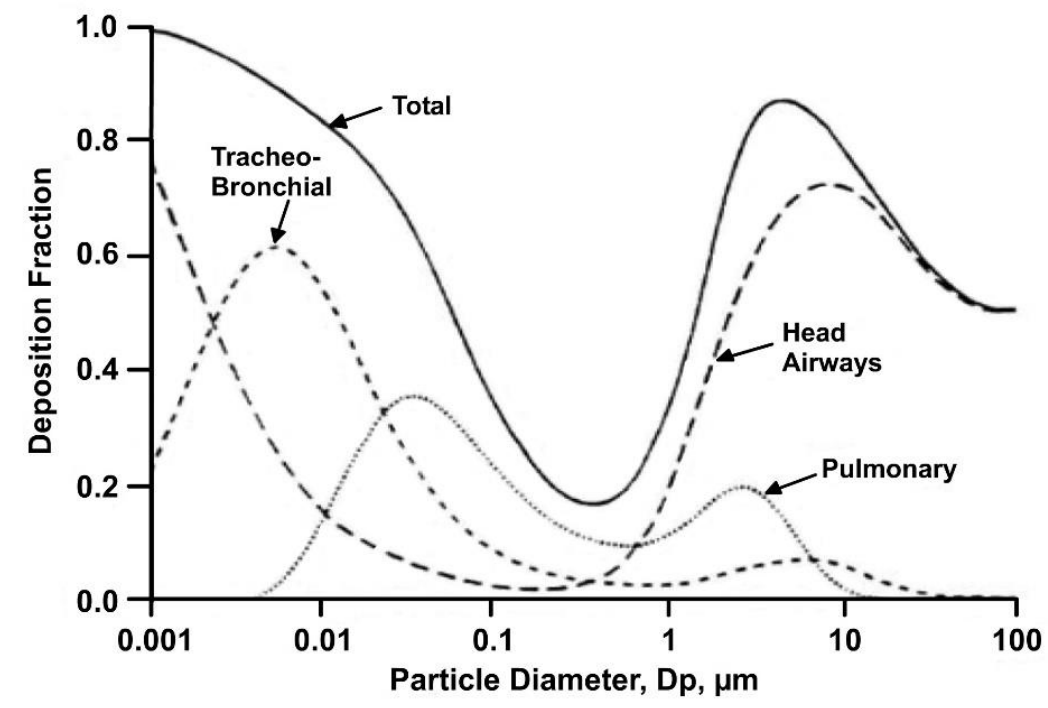

Fig. 4. The deposition location of inhaled particles according to their sizes [6]

There is some indication that metal particles can cause a variety of ailments, and this is summarized in Fig. 5.

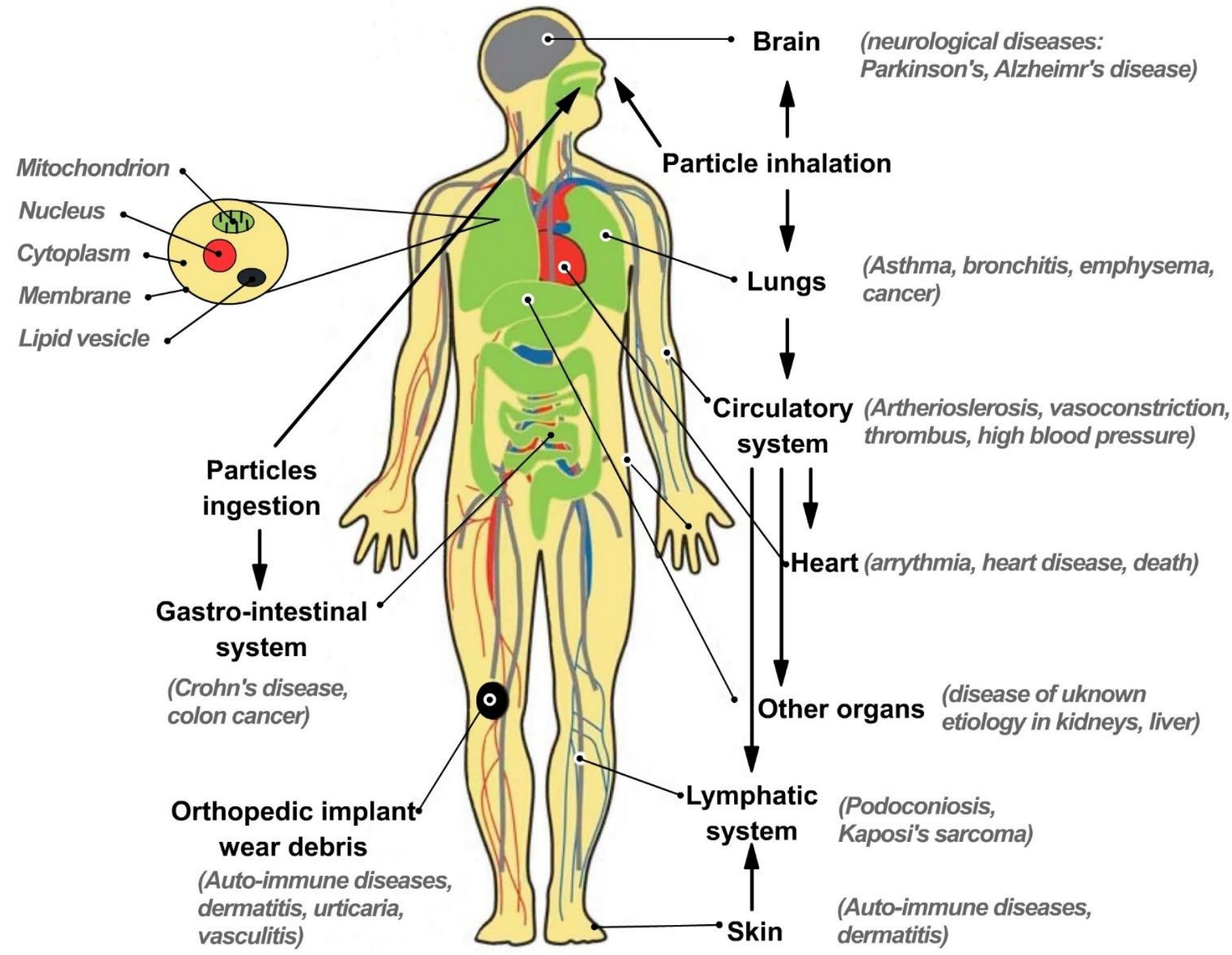

Fig. 5. Collation of the recorded diseases attributed to particles smaller than $1 \mu \mathrm{m}[6]$ 


\subsection{Inert gas asphyxiation}

Inert gases are used in laser metal AM machines to reduce the reactivity of the metal for processing purposes. Most metal AM machines either use Nitrogen or Argon. Inert gas asphyxiation is the main risk due to oxygen being displaced by either of these gases that have leaked for some reason. Since both gases are not detectable by humans, victims do not realize that they are inhaling air depleted of oxygen and as a result this can have a serious impact [4].

\subsection{Other hazards of AM technology}

In addition to the hazards mentioned above, the AM machine operator must be well aware of the following [7]:

- Hazard due to electrical power: The common hazard of injury from electricity due to improper use of the AM machine and all auxiliary equipment.

- Hazard due to laser radiation: Some of the equipment used in metal AM involves potentially hazardous energy sources such as lasers and electron beams, which could cause injury to eyes and skin.

- Thermal hazards: Hazard of injury due to hot machine components and parts at the end of the building process and during the cooling phase.

- Mechanical hazard: Risk of injury due to crushing or lifting of a heavy building platform

\section{Recommended protective equipment and precautions}

\subsection{Workplace protective equipment}

To ensure and increase the safety of the AM machine operators and personnel working in adjacent areas, it is necessary to equip the area with special equipment and safety features. Generally, to prevent and mitigate the hazards mentioned above, the workplace where the additive manufacturing takes place (i.e. not only place where the AM machine is located, but all places where interaction with the powder is expected) should be equipped above all with [1], [7], [4]:

- Advanced air filtration and dust extraction unit

- Wet vacuum cleaner (for immediate powder removal)

- Monitoring system for safe oxygen levels

- Efficient sealing from other areas

- Grounded electrical equipment

- Equipment with anti-spark design

- Non-flammable, cool and well-ventilated cabinet for powder barrel storage

- Fire extinguisher using special powder against burning metal

- Showers and eye wash fountain

- Ban of smoking, using naked flames and open sources of ignition

- Operating and safety rules (that workers must be trained in) including emergency procedures - above all description of first aid measures and firefighting measures.

\section{2. Personal protective equipment}

For workers' personal safety and elimination of basic hazards, it is essential to equip them with suitable protective equipment, which is dependent on the specific activities performed. Generally, at all times when handling the powder or working in places where the hazards above could be present, workers should wear [1], [7], [4]:

- A simple respirator (for short stay or less hazardous activities), full-face mask with particle filter or respiratory helmet with powered air purifying respirator (for direct interaction with powder and long-term stay in contaminated area); all respirators need to be based on P3 category filter.

- Closed protective clothing made of flame-retardant material; at best a non-textile (powder easily penetrates textile clothing and remains inside) disposable coverall (for the case of long-term powder handling or of dust swirling risk)

- Disposable nitrile protective gloves or heat-resistant gloves

- Safety glasses with side protection (in combination with simple respirator)

- Closed safety shoes with protection against ESD (electrostatic discharge) 


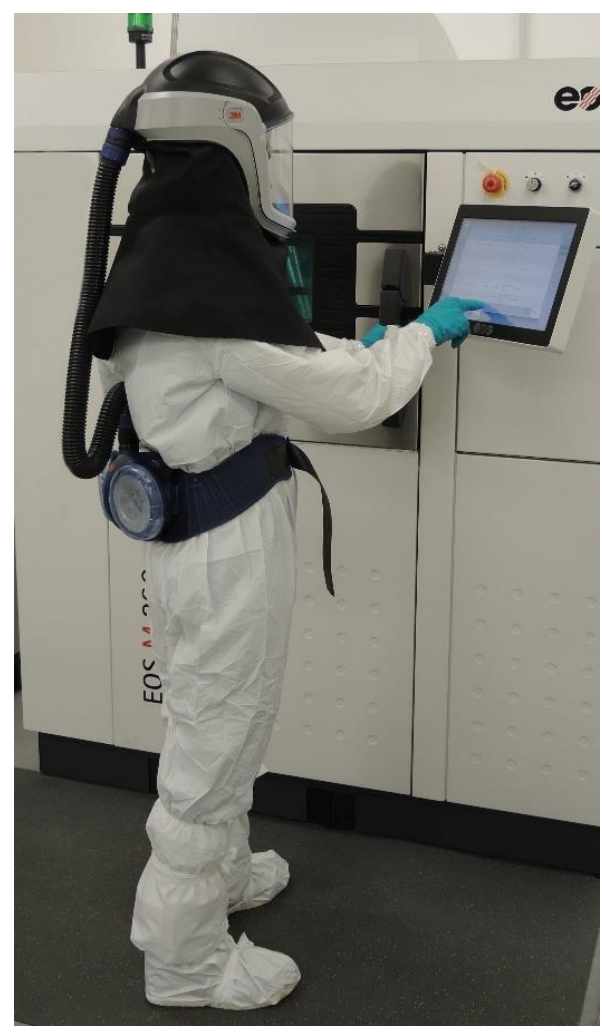

Fig. 6. Suitable protective equipment for direct interaction with the metal powder

\section{Conclusion}

Additive manufacturing is a modern manufacturing technology that has a number of advantages over conventional technologies and allows us to create complex components and things that cannot be produced in any other way. On the other hand, this technology also brings a number of new health hazards that threaten AM machine operators and people involved in the additive manufacturing process. These days, there are a lot of miscellaneous technologies on the market, each with their own specifics, benefits and hazards. These typical hazards include, besides hazards arising from handling powder feedstock materials, electrical energy hazards, irradiation hazards, entrapment, thermal hazards and others. In general, the greatest hazards arise from feedstock materials used; fine metal powder is especially dangerous.

This powder, which may have a wide variety of chemical compositions, is made up of particles - usually spheres with a diameter from 10 to $70 \mu \mathrm{m}$. However, even smaller particles may be present in this powder and other particles, not unfrequently of a diameter smaller than $1 \mu \mathrm{m}$, may occur in the AM machine process chamber as soot or metal condensate which arises during laser melting or the sintering process. At this size and under certain circumstances, metal powder can be prone to fire and explosion, and there is also the physiological concern of long-term inhalation or skin and eye contact with the metal powder or condensate. To minimize these hazards, workers have to obey instructions and safety rules (it is especially essential to avoid any ignition sources while handling powder or soot outside of the inert environment), and wear or use efficient personal protective equipment, above all with breathing-protection systems that filter out airborne powdered materials.

Along with development of personal protective equipment, safety and health-protective elements are also being developed and integrated into new AM machines and auxiliary equipment. The trend over recent years is to design machines where the operator practically does not come into immediate contact with the powder - e.g. many operations are performed automatically (e.g. powder sieving) and others take place in sealed work chambers to which the operator has access using special sleeves.

Despite all these measures, more research needs to be done to understand all the effects of fine powder on the human organism, to increase working safety and develop guaranteed effective personal protective equipment. As has been found, in accordance with the authors cited in this article, not all safety precautions and protective equipment are completely reliable, and metal powder handling could still pose a considerable health hazard - a face mask and most filtration units are unlikely to sufficiently prevent metal powder residue from entering the body; the powder can occur in unexpected places and nano-scale metal particles could penetrate the skin. So far it is too early to know what this might mean for humans at the cellular level and detailed toxicological investigations have not been conducted on all metal powders, and thus data is often limited to various ailments that have been reported by workers handling these powders in the long term or by workers from other industries. 
The necessary research that needs to be performed in collaboration with experts from different fields should answer the following questions:

- What effect does long-term exposure to metal powder have on the human organism?

- Which symptoms of a dangerous level of metal powder in the human body could be tested (i.e. during regular medical examinations)?

- What particles and what sizes of particles occur in the additive manufacturing process?

- How to recognize a place contaminated with dangerous particles?

- To what extent is current protective equipment effective? How to improve it?

\section{Acknowledgments}

The present contribution was supported from ERDF "Research of additive technologies for future applications in machinery industry - RTI plus" (No. CZ.02.1.01/0.0/0.0/18_069/0010040).

\section{References}

[1] Balakrishnan, V. N. (2019). Safety management in metal Additive Manufacturing: Observations from industry, Available from: issuu.com/inovar-communications/docs/mam_spring_2019_sp?e=32443561/68531372 Accessed: 2019-09-16

[2] Kowen, J. (2019). The challenges of metal powder removal: Managing risk, productivity and quality, Available from: issuu.com/inovar-communications/docs/mam_spring_2019_sp?e=32443561/68531372 Accessed: 2019-0916

[3] Tingskog, T. (2018). An introduction to metal powders for AM: Manufacturing processes and properties, Available from:issuu.com/inovar-communications/docs/mam_summer_2018_sp?e=32443561/63203285 Accessed:2019-0916

[4] Bhate, D. (2017). Installing a Metal 3D Printer: Part 3A (Safety: Risks), Available from: www.padtinc.com/blog/ installing-a-metal-3d-printer-part-3a-safety-risks/ Accessed: 2018-08-16

[5] Benson, J.M. (2012). Safety considerations when handling metal powders, Available from: www.saimm.co.za/ Journal/v112n07p563.pdf Accessed: 2018-08-16

[6] Buzea, C., Pacheo, I., Robbie, K. (2007). Nanomaterials and nanoparticles: Sources and toxicity, Biointerphases, vol. 2, Available from: arxiv.org/ftp/arxiv/papers/0801/0801.3280.pdf Accessed: 2019-09-16

[7] my.eos.info/\#\{1\} (2019). EOS GmbH, MS-MS1_Safety_data_sheet_5-0-0_06-15_en, Accessed: 2019-09-16

[8] www.lboro.ac.uk/research/amrg/about/ (2019). Loughborough University, Additive Manufacturing Research Group, The 7 Categories of Additive Manufacturing, Accessed: 2019-09-16

[9] www.astm.org/Standards/F2792 (2017). ASTM, Standard terminology for additive manufacturing, Accessed: 2017 09-01

[10] Nozar, M; Zetková, I; Hanzl, P \& Dana, M (2017). A Customer's View on Key Aspects of Metal Additive Manufacturing, Proceedings of the 28th DAAAM International Symposium, pp.957-966, B. Katalinic (Ed.), Published by DAAAM International, ISBN 978-3-902734-11-2, ISSN 1726-9679, Vienna, Austria; DOI: 10.2507/28th.daaam.proceedings. 133 